\title{
Intelligent Medical Decision System for Identifying Ultrasound Carotid Artery Images with Vascular Disease
}

\author{
N.Santhiyakumari, \\ Department of ECE, \\ K. S. Rangasamy College of Technology, \\ Tiruchengode-637215, \\ India.
}

\author{
M. Madheswaran \\ Center for Advance Research, Department of \\ Electronics and Communication Engineering, \\ Muthayammal Engineering College, \\ Rasipuram - 637408, India.
}

\begin{abstract}
The objective of this work is to develop and implement an intelligent medical decision system for identifying Ultrasound (US) carotid artery images with vascular diseases. The proposed method categorizes the carotid artery subjects into normal and diseased subjects' namely cerebrovascular and cardiovascular diseases. For each and every preprocessed ultrasound carotid artery image, contours are extracted using contour extraction techniques. Multilayer Back Propagation Network (MBPN) system has been developed for categorizing the carotid artery subjects. The obtained results show that MBPN system provides higher classification efficiency, with minimum training and testing time. It helps in developing Medical Decision System (MDS) for ultrasound carotid artery images. It can also be used as secondary observer in clinical decision making.
\end{abstract}

\section{Categories and Subject Descriptors G.4 MATLAB}

\section{General Terms}

Algorithm, Performance

\section{Keywords}

US carotid artery image, Contour extraction, Multilayer back propagation network, Neural network classifier, Medical decision system.

\section{INTRODUCTION}

The recent developments in medical image analysis have motivated advance research for developing a systematic approach for better interpretation. In recent past, advancements in the field of medical imaging have provided powerful noninvasive techniques to probe various pathologies of human body for better diagnosis. The accurate extraction of anatomical structures from the medical images becomes a challenging task for many clinical and research applications. The acquired ultrasound image is expected to have noise components which cannot be diagnosed with manual interpretations.
In the past century, the technologies have advanced due to the discovery of X-rays, Arteriography, Magnetic Resonance Imaging (MRI), Magnetic Resonance Angiography (MRA), Computed Tomography (CT), Positron Emission Tomography (PET), Single Photon Emission Computed Tomography (SPECT) and Ultrasonography. The basic idea of Medical Decision System (MDS) is to provide a computer based outputs for health care professionals and radiologist for diagnosing the subjects. The system can be considered as "second opinion" for improving the medical image interpretation for efficient diagnostic decision making process [1-5].

The advancements in medical imaging over the past few decades have enabled the physicians to non-invasively peer inside the human body for the purpose of diagnosis and therapy. With the advent of medical imaging modalities that provide different measures of internal anatomical structure and function, physicians are now able to perform typical clinical tasks such as patient diagnosis and monitoring. Successful development of Computed Aided Diagnosis (CAD) scheme makes the radiologist to investigate the nature of the subjects. Recently organs being subject to research for CAD include the kidney, brain, liver, colon, chest, breast, vascular system and skeletal system [6].

A novel automated method have been developed by Masahito Aoyama et al. [7] based on CAD scheme helps in assisting the diagnosis procedure for radiologists to make the difference between benign and malignant. In this technique database have been maintained for chest radiograph. Nodules were located with the help of radiologist. The resultant filter images from nodules were represented in a polar coordinate system. Analysis of contour lines of gray level distinction based on polar coordinate representation aids in segmenting the nodules automatically. In this, Linear Discriminant Analysis (LDA) procedures were used to select the clinical parameters namely age, sex and features. Cluster of features extracted from the analysis have been used as inputs for Artificial Neural Network (ANN). It was mentioned clearly that this method gave better results as compared to manual method. Ashizawa K et al. [8] suggested a new method which aids in distinguishing various interstitial lung diseases using an ANN. This network was designed in such a way that it differentiates among 11 interstitial lung diseases using 10 clinical parameters and 16 radiologic findings. It has been reported that ANN can provide differential 
diagnosis of interstitial lung disease using chest radiography and this reports were used as second opinion to assist radiologist.

Solid and sub solid pulmonary modules in CT images were automatically detected using model based CAD system was described by Colin C. McCulloch et al. [9]. In this technique multiple segmentation algorithms have been used to extract the noteworthy structures in the lungs. Throughout the lungs, probability of various anatomical events has been determined using bayesian statistical framework model. This CAD algorithm was used to measure the sensitivity improvement between two radiologists with respect to all non calcified nodules, solid and subsolid.

Classification of the hepatic lesions from CT images were proposed by Gletsos $\mathrm{M}$ et al. [10] using a CAD system. This system has two modules namely feature extraction and classification modules. The feature extraction module was used to calculate the average gray level and texture characteristics from the spatial gray-level co-occurrence matrices. This CAD system provides much appreciable classification efficiency in identifying different hepatic lesions using classifier module with three sequentially placed feed-forward neural networks.

Verikas A et al. [11] investigated an automated analysis of vocal cord images for diseases classification using CAD system. In this method, colour, texture, and geometrical features were used for obtaining a concise and informative representation of a vocal cord image. Pattern classifier was used in this system to categorize the images into healthy, diffuse and nodular classes analyzed data. Yoshida $\mathrm{H}$ et al. [12] developed a CAD system for CT Colonography (CTC). It also provides radiologists with a second opinion in an automated way to detect the locations of suspicious polyps and masses on CTC.

The review of literatures in the context of CAD explores its wide spread application in medical diagnosis. Each implementation uses different techniques and developed with an aim of identifying or classifying the pathology of interest. This indicates each CAD implementation is an application dependent. Similarly in the present work an effort has been taken to realize a MDM system for classifying three categories of carotid artery using US as imaging modality. The procedure formulated to develop a MDM system is specific and confined for US carotid artery image. The US carotid artery images may be diagnosed either subjectively or objectively. In subjective diagnosis, physicians based on their knowledge and expertise in the field interprets the content of carotid artery region and concludes the category. Obviously such decisions are susceptible to intra and inter expert variability, which makes diagnosis fallacious. An alternative may be the objective methods wherein the interpretations are made with assistance of computing procedures that are built on certain mathematical concepts. Such type of diagnosis offers consistent result and does not depend on the intra and inter experts' variability.

Ultrasound (US) imaging finds wide applications in the diagnosis of human vascular system due to its real time, noninvasive, non-radioactive and inexpensive nature [13 \& 14]. Besides the qualitative information for visual interpretation the quantitative information on the ultrasound scans, such as the sizes, edges and positions of anatomical structures, is also of significant interest for computer aided diagnosis. However the automatic analysis of ultrasound images is a difficult task because of the appearance of speckle patterns caused by the dephased echoes from the scatterers. Analysis of ultrasound images is complex due to the presence of speckle noise.

The speckle often obscures and masks diagnostically important features in US images and hence speckle reduction is a critical preprocessing step for feature extraction, analysis and recognition from medical US imagery measurements. This has initiated the researchers to develop better medical diagnosis modules and reliable decision making system. Medical Decision System (MDS) is being concentrated to use image processing concepts and neural network techniques to assist the physicians to take objective decisions during diagnosis. Although the carotid artery related diseases are extremely common in elderly subjects, these details are not adequately reported in medical image analysis hitherto [[15 \& 16].

The solution to develop such a MDS for identifying the normal and abnormal subjects is in high demand and to be addressed. In view of the above identified problem in human vascular structure, systematic investigations of ultrasound image capturing and post processing have been proposed in the present investigation.

Implementing such a MDS provides certain possibilities like i). Implement a MDS for analyzing and classifying US carotid artery images namely normal, cerebrovascular and cardiovascular subjects. ii). The realization of MDS by contour extraction techniques and neural network decision support system is in high demand and so far not been reported. iii) Establishing a quantitative protocol for ultrasound images. iv) Make comparative study on images for objective decision making. v) Develop an expert system that automatically recognizes the pathology or normality. vi) Implement image retrieval in medical application system. vii) Examine extent of healing or failure under post therapy observation [17].

The intention of the decision making system is to provide desired output based on the input it receives at the time of decision making. This depends on the way by which the system has been explored to the environment during the training phase with the assistance of thickness values. In the present study, decision making system using neural network classifier is developed. Multilayer Back Propagation Network (MBPN) architecture has been designed to classify the carotid artery category. The performance of the decision making module is analyzed in terms of classification efficiency, training and testing time for all the subjects thickness values as input.

In the present work, an attempt has been made for the first time to implement a MDS, that facilitates automated classification of US carotid artery images of three categories namely normal (NR), Cardiovascular diseases (CVD) and Cerebrovascualr diseases (CeVD). The prime focus of this paper is to realize a MDS with an intention to help the medical experts as secondary observer to perform unbiased diagnosis on US carotid artery images and to meet few possibilities of MDS system.

\section{MDS OF CAROTID IMAGES}

The MDS design requires three main areas for its implementation to detect a specific kind of abnormality. The structured procedure to realize a CCA medical diagnosis system is shown in Figure 1. The images acquired by using US scanning system are given as input to the devised MDS system. 


\subsection{Image data}

The images used for the analysis are acquired from two types of scanning systems namely,

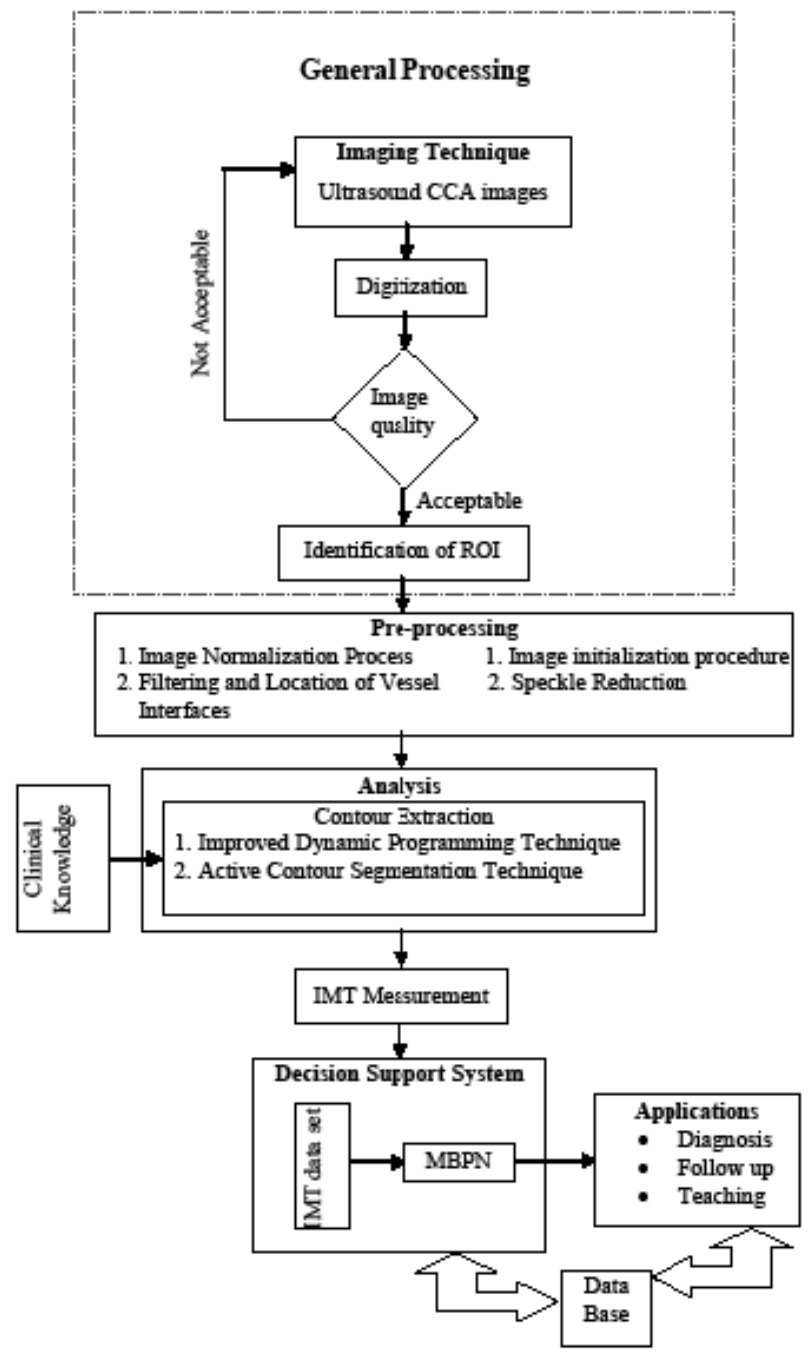

Figure 1. Proposed MDS for US Carotid artery

i) Aloka Prosound Alpha -10 (SSD $\alpha-10$, Model No-M00720 Japan) ultrasound scanner with a high resolution and digital beam former with ultimate compound technology has been used for the acquisition of the images. The built-in compound pulse wave generator facilitates to optimize the contrast and to obtain an enhanced focus and spatial resolution. A multifrequency linear transducer with a frequency range of $5-10 \mathrm{MHz}$ has been used for recording the arterial movements. The transducer is operated at a frequency of $7.5 \mathrm{MHz}$ to obtain the arterial movements and the movements are recorded using video recorder.

ii) Philips HD11XE US machine (Model No - HDI5000 sonoct) with the broadband compact linear array transducer has been used for the data acquisition of the images. High definition sonoct has been used to capture the real time compound by using transmit beam steering techniques. A multifrequency linear transducer with a frequency range of 7-15 MHz has been used for recording the arterial movements. The transducer is operated at a frequency of $12 \mathrm{MHz}$ to obtain the arterial movements and the movements are recorded using video recorder.

As the sonographic evaluation is made based on the distribution of echogenity that reflects tissue characteristics, for better echo visualization. The transverse and longitudinal section of carotid artery is taken to include normal and abnormal subjects as suggested by the experts. This also ensures better visual interpretation of the normal and diseased carotid artery. The exact transducer position (at right angles to adventitia) can be identified using transducer by placing it at different inclined angles. The video is recorded for a period of 10 seconds for each subject to show the transverse and longitudinal sections of the CCA. The transducer is placed exactly at the starting point of the bifurcation of CCA to obtain an uniform lumen for different subjects. Thus the left end of the image indicates the starting point of bifurcation.

The recorded video is converted into frames employing video decompiler. The processed frames are stored as still images in a computing system for further processing. In total, 100 images with 50 images in each category are obtained from male and female subjects of age $20-70$ years for training and 50 images in each category is acquired under the same age group for testing. The carotid images of normal and abnormal are considered for the analysis. The necessary clinical informations of the training images are documented. To make the testing process unbiased these details are veiled for the test images.

\subsection{Pre-processing and Contour Extraction}

In order to preserve the carotid artery region from the overall tissue characteristics, the following preprocessing procedure has been done prior to the contour extraction technique [18].

Step1: Identification of the region of interest from the acquired images.

Step2: Intensity normalization using average intensity histogram.

Step3: Horizontal smooth filtering to remove echo dropouts in US images.

Step4: Blurring to locate the vessel interfaces.

After the preprocessing steps, the arterial layers have been extracted using Improved Dynamic Programming (IDP) Algorithm [19]. In Active Contour (AC) extraction method, the acquired images have been preprocessed using speckle reducing anisotropic diffusion technique [20] from the initialized region [21]. After speckle reduction, the contours have been extracted using energy minimization process [22]. The thickness values obtained from two contour extraction techniques have been used as input parameters for neural network classifier.

\subsection{Neural Network Classifier}

Normally, Neural Network based solutions are developed in four steps. They are

1. Data transformation, scaling or normalization.

2. Network architecture definition.

3. Learning algorithm. 
4.

Validation step.

First Step: In this step, large variation in the input data can slow down or even prevent the training of the network. To overcome this potential problem, the data are usually scaled using statistical, min-max, sigmoidal or principal component transformations [23]. It is also important that absolute input values are scaled to avoid asymptotic issues [24].

Second Step: In this step, the number of hidden layers, the number of neurons in each layer and the connectivity between the neurons are set. There are three basic layers or levels of data processing units namely, the input layer, the hidden layer and the output layer. Each of these layers consists of processing neurons. The number of input neurons, output neurons and the neurons in the hidden layer depend upon the problem being studied. If the number of neurons in the hidden layer is small, the network may not have sufficient degrees of freedom to learn the process correctly. If the number is too high, the training will take a long time and the network may sometimes over-fit the data [25]. Validation set error is often used to determine the optimal number of hidden neurons for a given study.

Third Step: In this step, a learning algorithm is used to train the network to respond correctly to a given set of inputs. The training goal is to find parameters that result in the best performance of the network with unfamiliar data. An ANN is better trained when more input data is available. Through training it is possible to determine ANN weights. At the beginning of training, the initial value of weights can be assigned randomly or based on experience. In the process, the learning algorithm systematically changes the weights to perform a desired input-output association correctly. Many methods exist for training, and the process of learning is terminated when the difference between the ANN output and the actual output is small. At this stage, the ANN is considered trained.

Fourth Step: At the end, the validation step in which the performance of the trained ANN model is tested through some selected validation set data. Validation can be employed to monitor the network error during training to determine the optimal number of training iterations. It can also be used to find out the optimal number of hidden neurons.

\subsection{Multilayer Back Propagation Network (MBPN)}

The back propagation learning procedure has become the single most popular method to train networks. It has been used to train networks in problem domains including character recognition, speech recognition, sonar detection and many more. The MBPN architecture consists of a network of nodes organized in layers. A typical MBPN network consists of three or more layers of nodes: an input layer that receives external inputs, one or more hidden layers, and an output layer which generates the classification results. Note that unlike other layers, no computation is involved in the input layer. The principle of the network is that when data are presented at the input layer, the network nodes run calculations in the consecutive layers until an output value is obtained at each of the output nodes. This output will indicate the appropriate class for the input data.

\section{Back Propagation Algorithm (BPA)}

The algorithm is developed considering supervised method for measuring minimum error value and steepest-descent method to examine a global minimum. The flow-chart for the algorithm is given in Fig 2.

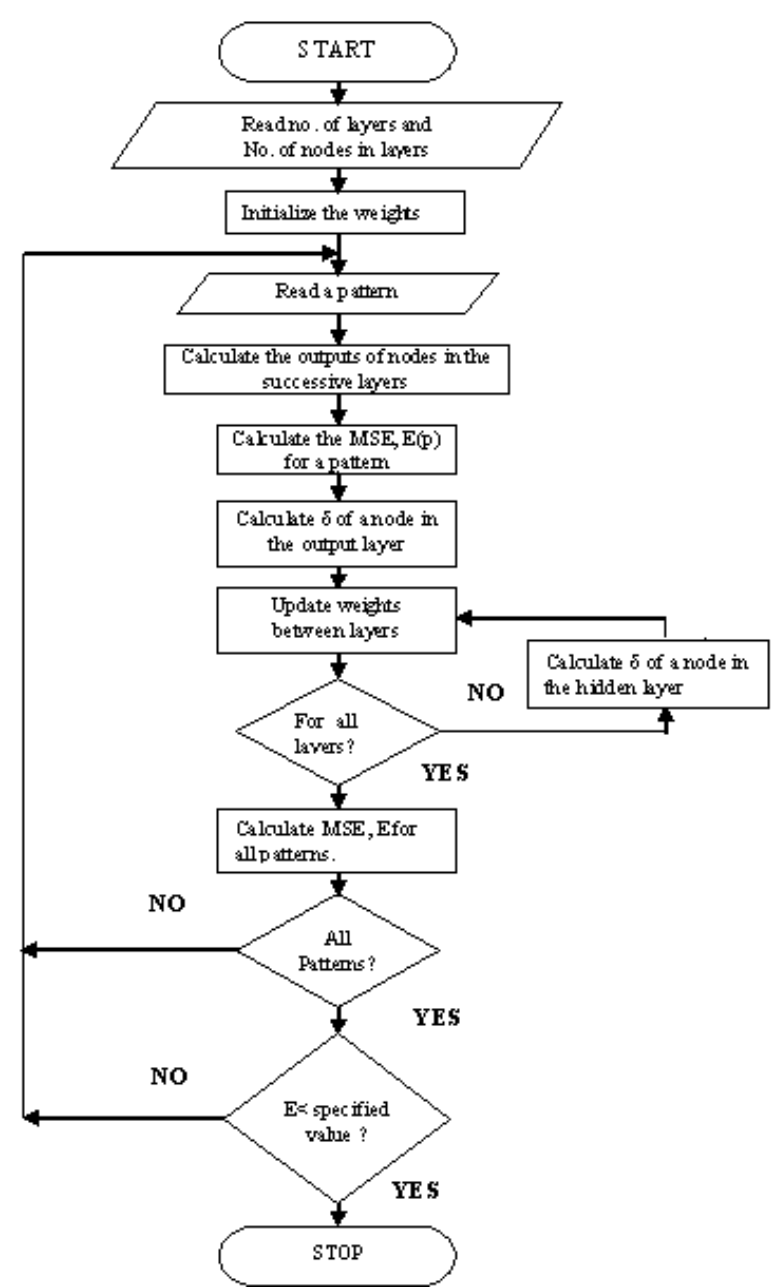

Figure 2. Flowchart for backpropagation algorithm

Training

The training process requires a set of examples of proper network behavior that includes network inputs and target outputs. During the training, the weights and biases of the network are adjusted iteratively to optimize the network performance function. The performance function for the networks is Mean Square Error (MSE)-the average squared error between the network output and the target output. There are generally five steps in the training process:

Step 1. Preprocess data to produce the most efficient training, it is helpful to preprocess the data before training. This means to scale the inputs and targets data to ensure they always fall within a specified range.

Step 2. Initialize the weight values. Before training a network, the weights and biases must be initialized. In order to 
avoid the result located in flat area, random weight values are selected.

Step 3. For input sample, calculate outputs of arbitrary node in both hidden layer and output layer (in forward propagation).

Step 4. Calculate errors of hidden layer and output layer (in backward propagation).

Step 5. Adjust weight values according to error function until the minimal error is achieved. After training the weights are fixed and can be used for practical classification.

MBPN learning process depends on three parameters namely learning coefficient $(\eta)$, momentum $(\beta)$ and mean square error $\left(\delta_{\max }\right)$. Learning coefficient $(\eta)$ determines the size of the weight changes. Its useful range is between 0.05 and 2 and depends on the problem. Momentum $(\beta)$ causes the weight changes to be dependent on more than one input pattern. The change is a linear combination of the current gradient and previous gradient. The useful range for this parameter is between 0 and 1 . Mean square error $\left(\delta_{\max }\right)$ is the parameter where the network error dips below a particular error threshold. A threshold of 0.001 squared errors is usually desirable.

MBPN is trained with the derived layer thickness values irrespective of the significant level and correlation between the categories. The network learning parameters, selection of number of hidden layers and hidden layer neurons are optimized to yield better classification efficiency.

\section{RESULTS AND DISCUSSION}

Carotid artery is categorised based on the output of the decision making system. Consequently it is sufficient to have one neuron in the output layer. The results obtained by varying the parameters like learning rate, momentum, threshold value are discussed and presented here.

\subsection{Learning rate}

The performance of MBPN for various learning rates $(\eta)$ in the range of 0.1-1.0 with an incremental value of 0.1 is shown in Table 1. The classification efficiency, training and testing time are measured for each value of ' $\eta$ '. The training is made with the layer thickness extracted from 50 images in each CCA category and to test the MBPN, same image data set is used. The results obtained shows that the training time changes between 1.94 and 1.34 for increase in ' $\eta$ ' value. For Normal (NR) images, maximum classification efficiency of $96 \%$ is achieved for $\eta=0.6$ and minimum of $68 \%$ for $\eta=1$. In case of Cerebrovascular Disease (CeVD) images, for the same value of ' $\eta$ ', the maximum classification efficiency obtained is $90 \%$ which is lesser than NR and no change in minimum value is noticed. The classification efficiency varies from $64 \%$ to $92 \%$ for Cardiovascular Disease (CVD), with the maximum classification efficiency of $92 \%$ that results for $\eta=0.6$. To select the appropriate and optimum value, it can be seen that for $\eta=$ 0.6 the classification efficiency is maximum for all three CCA category. Even though the training time is slightly higher by 0.13 seconds than the overall average training time of 1.73 seconds, as the classification efficiency is better, the ' $\eta$ ' is fixed at 0.6 . The average testing time achieved for $\eta=0.6$ is 0.0130 seconds.

Table 1. Training time and classification efficiency for various learning rate $(\eta)$

\begin{tabular}{|c|c|c|c|c|c|c|}
\hline \multirow[b]{2}{*}{ S.No } & \multirow{2}{*}{$\begin{array}{l}\text { Learn } \\
\text { ing } \\
\text { Rate } \\
(\eta)\end{array}$} & \multirow{2}{*}{$\begin{array}{c}\text { Average } \\
\text { Training } \\
\text { Time } \\
\text { (in } \\
\text { seconds) }\end{array}$} & \multirow{2}{*}{$\begin{array}{c}\text { Average } \\
\text { Testing } \\
\text { Time } \\
\text { (in } \\
\text { seconds) }\end{array}$} & \multicolumn{3}{|c|}{$\begin{array}{c}\text { Classification } \\
\text { Efficiency (\%) }\end{array}$} \\
\hline & & & & NR & $\begin{array}{l}\mathrm{CeV} \\
\text { D }\end{array}$ & $\begin{array}{l}\text { C } \\
\text { V } \\
\text { D }\end{array}$ \\
\hline 1 & 0.1 & 1.94 & 0.0163 & 79 & 82 & 80 \\
\hline 2 & 0.2 & 1.92 & 0.0159 & 78 & 81 & 80 \\
\hline 3 & 0.3 & 1.90 & 0.0150 & 78 & 76 & 82 \\
\hline 4 & 0.4 & 1.88 & 0.0146 & 82 & 80 & 85 \\
\hline 5 & 0.5 & 1.78 & 0.0138 & 89 & 85 & 88 \\
\hline 6 & 0.6 & 1.86 & 0.0130 & 96 & 90 & 92 \\
\hline 7 & 0.7 & 1.67 & 0.0126 & 82 & 84 & 78 \\
\hline 8 & 0.8 & 1.56 & 0.0124 & 75 & 76 & 74 \\
\hline 9 & 0.9 & 1.45 & 0.0120 & 70 & 74 & 68 \\
\hline 10 & 1.0 & 1.34 & 0.0115 & 68 & 72 & 64 \\
\hline
\end{tabular}

Training time is estimated for 50 training images in each CCA category and same image data set is used as a test image to evaluate classification efficiency. The activation function LOGSIG at hidden layer and PURELIN at output layer is fixed. Maximum number of iterations required has been found to be 100000.

\subsection{Momentum}

The functioning of MBPN for various values of momentum $(\beta)$ is given in Table 2. The value is varied between 0.001 and 0.01 in steps of 0.001 . The classification efficiency, training and testing times are measured for each value of ' $\beta$ '. Here also the training is made separately with layer thickness values extracted from 50 contour images in each category and to test the network same image data set is used. It can be seen from Table 4 , the best training time of 1.80 is achieved for $\beta=0.004$. This provides the classification efficiency of $96 \%$ for NR, $90 \%$ for CeVD and $92 \%$ for CVD. The average testing time is higher than the few other cases, but it is almost the same as obtained with ' $\eta$ '. For $\beta=0.006$, though the classification efficiency is same as before, the time taken by MBPN to get trained is more. In the remaining cases, both training and testing time for classification efficiency is not favourable. Therefore the optimum value $\beta=0.004$ is assigned in MBPN architecture.

Table 2. Training time and classification efficiency for various Momentum ( $\beta$ )

\begin{tabular}{|c|c|c|c|c|c|c|}
\hline \multirow[b]{2}{*}{ S.No } & \multirow{2}{*}{$\begin{array}{c}\text { Mome } \\
\text { ntum }\end{array}$} & \multirow{2}{*}{$\begin{array}{c}\text { Average } \\
\text { Training } \\
\text { Time } \\
\text { (in } \\
\text { seconds) }\end{array}$} & \multirow{2}{*}{$\begin{array}{c}\text { Average } \\
\text { Testing } \\
\text { Time } \\
\text { (in } \\
\text { seconds) }\end{array}$} & \multicolumn{3}{|c|}{$\begin{array}{c}\text { Classification } \\
\text { Efficiency (\%) }\end{array}$} \\
\hline & & & & NR & $\begin{array}{l}\mathrm{CeV} \\
\text { D }\end{array}$ & $\begin{array}{l}\text { C } \\
\text { V } \\
\text { D }\end{array}$ \\
\hline 1 & 0.001 & 1.98 & 0.0156 & 82 & 84 & 86 \\
\hline
\end{tabular}




\begin{tabular}{|c|c|c|c|c|c|c|}
\hline 2 & 0.002 & 1.74 & 0.0148 & 86 & 85 & 84 \\
\hline 3 & 0.003 & 1.91 & 0.0150 & 80 & 78 & 85 \\
\hline $\mathbf{4}$ & $\mathbf{0 . 0 0 4}$ & $\mathbf{1 . 8 0}$ & $\mathbf{0 . 0 1 2 9}$ & $\mathbf{9 6}$ & $\mathbf{9 0}$ & $\mathbf{9 2}$ \\
\hline 5 & 0.005 & 1.77 & 0.0146 & 89 & 85 & 88 \\
\hline 6 & 0.006 & 2.34 & 0.0152 & 96 & 90 & 92 \\
\hline 7 & 0.007 & 1.88 & 0.0136 & 82 & 84 & 86 \\
\hline 8 & 0.008 & 2.63 & 0.0134 & 78 & 80 & 83 \\
\hline 9 & 0.009 & 1.56 & 0.0110 & 81 & 80 & 84 \\
\hline 10 & 0.01 & 1.66 & 0.0112 & 78 & 84 & 82 \\
\hline
\end{tabular}

Training time is estimated for 50 training images in each CCA category and same image data set is used as a test image to evaluate classification efficiency. The activation function LOGSIG for the hidden layer and PURELIN for the output layer is fixed. Maximum number of iterations required has been found to be 100000 .

\subsection{Threshold value}

The ultimate inference regarding the category also depends on the threshold value fixed at the output units. The performance of MBPN for different threshold value settings is studied by incrementing its value by 0.05 from 0.1 to 1.0 . The classification efficiency is noted for each setting and best possible threshold value is assigned. The results obtained shows for the value in the range $0.40 \mathrm{~mm}-0.88 \mathrm{~mm}$; the NR images are identified correctly with the classification efficiency of $96 \%$. The CeVD and CVD images are also categorized, but with the classification efficiency of $70 \%$ and $76 \%$ respectively. For values from $1 \mathrm{~mm}$ to $2.5 \mathrm{~mm}$, CeVD images are discriminated with the classification efficiency of $90 \%$, while NR and CVD with $78 \%$ and $64 \%$.

The CVD images are correctly identified for the value $>2.5 \mathrm{~mm}$ and the classification efficiency obtained is $92 \%$. For this threshold value, in case of $\mathrm{NR}$ and CeVD, the classification efficiency achieved is $64 \%$ and $60 \%$ based on this study, the threshold value is fixed to make decision on given input image. The input image is determined as NR category if the achieved output value of MBPN is $\leq 1.0 \mathrm{~mm}$. If the value is $\geq 1.0 \mathrm{~mm}$ and $\leq 2.5 \mathrm{~mm}$ then the decision on input image is made as CeVD. If the value is greater than $2.5 \mathrm{~mm}$, then the input is assessed as CVD category.

\subsection{Activation function}

Different activation functions are available in the neural networks. In this study LOGSIG for the hidden layer and PURELIN for the output layer have been chosen. Similar to the procedure followed for fixing $\eta, \beta$ and $T_{h}$, the training is made with the layer thickness values extracted from 50 contour images in each category and same image data set is used to test the performance of the network.

\subsection{Hidden layer}

As mentioned the choice of number of hidden layer and the number of hidden nodes per layer is arbitrary. But to have systematic approach, one hidden layer is selected initially with one node and increased by one upon estimating training, testing and classification efficiency. During this process if the classification efficiency drops or training or testing times increase by increasing the number nodes per layer or the hidden layer, no further modification is made.

The Table 3 shows the results obtained in terms of classification efficiency, training and testing time. The performance goal of the network which is quantified with the parameter mean square error is assigned as 0.001 . For the hidden nodes upto to 30 , the performance goal is not met. Even though it is possible to achieve the goal for the number of hidden units 35 to 55 , maximum classification efficiency and minimum training and testing times are not achieved unless the number of hidden nodes is 50. Therefore for present application it has been decided to fix the number of hidden layer as one and number of hidden units in that layer as 50 .

Table 4 gives the entire details of MBPN parameters which are selected based on the above results. It is used for implementing the MDM system. Such settings offer the classification efficiency of $96 \%, 90 \%$ and $92 \%$ for NR, CeVD and CVD respectively.

Table 3. Training time and classification efficiency for various hidden units

\begin{tabular}{|c|c|c|c|c|c|c|}
\hline \multirow[b]{2}{*}{$\begin{array}{l}\text { No. of } \\
\text { neuron } \\
\text { s in the } \\
\text { hidden } \\
\text { layer }\end{array}$} & \multirow[b]{2}{*}{$\begin{array}{c}\text { Training } \\
\text { status }\end{array}$} & \multirow{2}{*}{$\begin{array}{l}\text { Avera } \\
\text { ge } \\
\text { Traini } \\
\text { ng } \\
\text { Time } \\
\text { (in } \\
\text { secon } \\
\text { ds) }\end{array}$} & \multirow{2}{*}{$\begin{array}{c}\text { Averag } \\
\text { e } \\
\text { Testing } \\
\text { Time } \\
\text { (in } \\
\text { seconds } \\
\text { ) }\end{array}$} & \multicolumn{3}{|c|}{$\begin{array}{l}\text { Classification } \\
\text { Efficiency (\%) }\end{array}$} \\
\hline & & & & $\begin{array}{l}\mathbf{N} \\
\mathbf{R}\end{array}$ & $\begin{array}{l}\mathrm{CeV} \\
\text { D }\end{array}$ & $\begin{array}{l}\text { C } \\
\text { V } \\
\text { D }\end{array}$ \\
\hline 5 & $\begin{array}{l}\text { goal not } \\
\text { met }\end{array}$ & - & - & - & - & - \\
\hline 10 & $\begin{array}{l}\text { goal not } \\
\text { met }\end{array}$ & - & - & - & - & - \\
\hline 15 & $\begin{array}{l}\text { goal not } \\
\text { met }\end{array}$ & - & - & - & - & - \\
\hline 20 & $\begin{array}{c}\text { goal not } \\
\text { met }\end{array}$ & - & - & - & - & - \\
\hline 25 & $\begin{array}{c}\text { goal not } \\
\text { met }\end{array}$ & - & - & - & - & - \\
\hline 30 & $\begin{array}{c}\text { goal not } \\
\text { met }\end{array}$ & - & - & - & - & - \\
\hline 35 & goal met & 1.925 & 0.0195 & 70 & 68 & 70 \\
\hline 40 & goal met & 1.765 & 0.0186 & 74 & 70 & 72 \\
\hline 45 & goal met & 1.542 & 0.0170 & 78 & 72 & 76 \\
\hline 47 & goal met & 1.431 & 0.0165 & 82 & 76 & 84 \\
\hline 49 & goal met & 1.322 & 0.0154 & 85 & 82 & 89 \\
\hline 50 & goal met & 1.285 & 0.0132 & 96 & 90 & 92 \\
\hline
\end{tabular}




\begin{tabular}{|c|c|c|c|c|c|c|}
\hline 51 & goal met & 1.474 & 0.0121 & 87 & 84 & 86 \\
\hline 53 & goal met & 1.678 & 0.0151 & 80 & 82 & 78 \\
\hline 55 & goal met & 1.846 & 0.0196 & 74 & 80 & 76 \\
\hline
\end{tabular}

Training time is estimated for 50 training images in each CCA category and same image data set is used as a test image to evaluate classification efficiency. Mean square error $=0.001$. Maximum number of iterations required has been found to be 100000 .

Table 4. Details of MBPN architecture parameters fixed for MBPN - ANN

\begin{tabular}{|l|l|l|}
\hline S.No. & $\begin{array}{l}\text { MBPN architectural } \\
\text { issues }\end{array}$ & Selected design factors \\
\hline 1. & Learning Rate $\eta)$ & 0.6 \\
\hline 2. & Momentum $(\beta)$ & 0.004 \\
\hline 3. & Threshold Value $\left(\mathrm{T}_{\mathrm{h}}\right)$ & $\begin{array}{l}\text { MBPN }- \text { ANN : } \\
\leq 1 \mathrm{~mm} \text { for NR; } \\
>1 \mathrm{~mm} \text { and } \leq 2.5 \mathrm{~mm} \\
\text { for CeVD; } \\
>2.5 \mathrm{~mm} \text { for CVD. }\end{array}$ \\
\hline 4. & Activation function & $\begin{array}{l}\text { LOGSIG at the hidden } \\
\text { layer and PURELIN at } \\
\text { the output layer }\end{array}$ \\
\hline 5. & Hidden layer & 1 \\
\hline 6. & Number of hidden units & 50 \\
\hline 7. & Input neurons & 50 \\
\hline 8. & Output neurons & 1 \\
\hline 9. & Mean Square Error $(\delta)$ & 0.001 \\
\hline 10. & Number of iterations & 100000 \\
\hline
\end{tabular}

\subsection{Performance of Medical Decision System}

The initial requirements to design MBPN are fulfilled by making meticulous study on architectural issues of the network. The appropriate factors are designed and formulated accordingly. Now with the available decision making system the input images of unknown category have to be identified. Reliable and proficient MDM system has been realized based on the classification efficiencies of US CCA images.

Input training patterns for various age groups are decided in this study. Fifty inputs are taken for training the network and remaining are taken as test patterns. The output displayed by the test network is 'Normal subject' if the layer thickness values are less than $1 \mathrm{~mm}$. If the thickness is more due to the pathologies developed or the thickness is more due to the abnormalities of the vessel, then the output displayed as 'Abnormal subject', such subject has to Meet the physicians for further diagnosis and treatment. Thus this study has recorded the outputs obtained for both the normal and abnormal subjects.

\section{CONCLUSION}

An Intelligent medical decision system for identifying Ultrasound (US) carotid artery images with vascular diseases have been developed and implemented. Using suitable preprocessing procedure, pixels of carotid artery regions are retained which facilitate to extract the contours of the arterial layers. The study reveals that the MBPN based MDS developed offers higher classification and actual efficiency. It is described in performance evaluation measure. As the input images are quantified by numerical value it is possible to create a universal reference for all three categories, make comparative study on values obtained to decide on category and know the extent of pathology involved. It is also believed the proposed MDS may assist the physicians to predict future possibility of normal subject becoming abnormal based on intima media thickness value. Such a MDS helps the physician to study extensively the characteristics of carotid artery more reliably, which finds broad clinical application.

\section{REFERENCES}

[1] Troccaz, J., Baumann, M., Berkelman, P., Cinquin, P., Daanen, V., Leroy, A., Marchal, M., Payan, Y., Promayon, E., Voros, S., Bart, S., Bolla, M., Chartier- Kastler, E., Descotes, J.-L., Dusserre, A., Giraud, J.-Y., Long, J.-A., Moalic, R., Mozer, P., 2006. Medical image computing and computer-aided medical interventions applied to soft tissues: Work in progress in urology. Proc. IEEE 94(9):1665- 1677.

[2] Summers, R. M., 2003. Road maps for advancement of radiologic computer- aided detection in the 21 st century. Radiology 229: 11- 13.

[3] Maryellen, L., Giger, N. K., Armato, S. G., 2001. Computer-aided diagnosis in medical imaging. IEEE Trans. Med. Imag. 20 (12):1205-1208.

[4] Doi, K., 2005. Current status and future potential of computer-aided diagnosis in medical imaging. Brit. J. Radiol. 78:S3-S19.

[5] Erickson, B. J., Bartholmai, B., 2002. Computer-aided detection and diagnosis at the start of the third millennium. J. Digit. Imaging 15:59-68.

[6] Bommanna Raja, K., Madheswaran, M., Thyagarajah, K., 2008. A Hybrid Fuzzy- Neural system for Computer Aided Diagnosis of Ultrasound Kidney Images Using Prominent Features. J. Med. Syst. 32: 65-83.

[7] Aoyama, M., Li, Q., Katsuragawa, S., MacMahon, H., Doi, K., 2002. Automated computerized scheme for distinction between benign and malignant solitary pulmonary nodules on chest images. Med. Phys. 29(5):701-708.

[8] Arimura, H., Katsuragawa, S., Suzuki, K., Li, F., Shiraishi, J., Sone, S., Doi, K., 2004. Computerized scheme for automated detection of lung nodules in low-dose computed tomography images for lung cancer screening. Acad. Radiol. 11(6):617-629.

[9] McCulloch, C. C., Kaucic, R. A., Mendonça, P. R. S., Walter, D. J., Avila, R. S., 2004. Model-based detection of lung nodules in computed tomography exams: thoracic computer-aided diagnosis. Acad. Radiol. 11(3):258-266. 
[10] Gletsos, M., Mougiakakou, S. G., Matsopoulos, G. K., Nikita, K. S., Nikita, A. S., Kelekis, D., 2003. A computeraided diagnostic system to characterize $\mathrm{CT}$ focal liver lesions: design and optimization of a neural network classifier. IEEE Trans. Inf. Technol. Biomed. 7 (3):153162.

[11] Verikas, A., Gelzinis, A., Bacauskiene, M., Uloza, V., 2006.Towards a computer aided diagnosis system for vocal cord diseases. Artif. Intell. Med. 36:71- 84.

[12] Yoshida, H., Dachman, A. H., 2005. CAD techniques, challenges and controversies in CT colonography. J. Abdom. Imaging 30:24-39.

[13] Jegelevicius D. and Lukosevicius A. 2002.Ultrasonic measurements of human carotid artery wall intima-media Thickness. Ultragarsas. 43-47.

[14] Pierre-Jean Touboul. Clinical impact of intima media measurement. European Journal of Ultrasound. 16.105 $113,2002$.

[15] Huang, S.-F., Chang, R.-F., Chen, D.-R., Moon, W. K., 2004. Charac-terization of speculation on ultrasound lesions. IEEE Trans. Med. Imag. 23(1):111- 121.

[16] Loizou, C. P., Christodoulou, C., Pattischis, C. S., Istepanian, R. H., Pantziaris, M., Nicolaides, A., 2002. Speckle reduction in ultrasound images of atherosclerotic carotid plaque. IEEE Proc. 14th Intl. Conf. Digital Signal Processing. Santorini, Greece, 1:525-528.

[17] Santhiyakumari N., Madheswaran M. 2008. Medical decision making system using intima media thickness measurement. Proceedings of Fifth International Conference on Medical Informatics and Telemedicine Conference.1.

[18] Santhiyakumari N., Madheswaran M. 2006. Estimation of layer thickness of arterio carotis using Dynamic
Programmin Procedure. Proceedings of third Cairo International Biomedical Engineering Conference. IP2- 4. $1-4$.

[19] Santhiyakumari N., Madheswaran M. 2008. Non-Invasive Evaluation of carotid artery wall thickness using improved dynamic programming technique. Journal of Signal, Image and Video processing (Springer). 2. 183-193.

[20] Santhiyakumari N., Madheswaran M. 2007. Extraction of Intima- Media Layer of Arteria- Carotis and Evaluation of its thickness using Active contour approach. Proceedings of International Conference on intelligent and advanced systems. IP_MS1. 582-586.

[21] Santhiyakumari N., Madheswaran M. 2008. Analysis of Atherosclerosis for identification of Cerebrovascular and Cardiovascular Diseases using Active Contour Segmentation of Carotid Artery. Proceedings of International Symposium on Global Trends in Bio Medical Informatics Research, Education and Commercialization. 1. 40.

[22] Santhiyakumari N., Madheswaran M. 2009. Analysis of atherosclerosis for identification of cerebrovascular and cardiovascular diseases using active contour segmentation of carotid artery', International Journal of Biomedical Engineering and consumer health Informatics. 1(2). 121125.

[23] Priddy L.K. and Keller E.P. 2005. Artificial neural networks an Introduction. SPIE Press. Bellingham. Washington.

[24] Haykin S. 1994. Neural networks. Macmillan College Publishing Company. Englewood Cliffs. NJ.

[25] Karunanithi N., Grenney W.J., Whitley D. and Bovee K. 1994. Neural Networks for River Flow Prediction. J. Computing in Civil Eng. ASCE. 8(2). 201-220. 